\title{
Estrategias competitivas para fortalecer sectores de actividad empresarial en el mercado global
}

\author{
Narváez, Mercy* \\ Fernández, Gladys**
}

\section{Resumen}

El actual modelo económico, en el cual la apertura comercial y el flujo de intercambio de productos y servicios es cada vez más internacionalizado, exige a las empresas y a los sectores donde ellas radican enfrentar nuevos desafíos y adaptarse a los cambios que tal situación supone. De allí que este artículo pretenda identificar algunas estrategias competitivas que permitan a los sectores de actividad empresarial obtener una posición ventajosa en el entramado mundial. Partiendo de un análisis descriptivo fundamentado en una contrastación teórica, se desprende de este estudio que para alcanzar una posición competitiva en el mercado global se deben establecer nuevos esquemas de actuación, tales como: conducta de integración, desarrollo de capacidades medulares, cooperación con entes externos, especialización, solidaridad y contribución social.

Palabras clave: Estrategias competitivas, mercado global, sectores de actividad empresarial, competitividad.

\section{Competitive Strategies to Strengthen Managerial Activity Sectors in the Global Market}

\section{Abstract}

The current economic model, in which commercial openness and the flow of product and service exchange is increasingly internationalized, demands that companies and the sectors where

Recibido: 23-01-07. Aceptado: 15-07-07

* $\quad$ Lic. en Administración. Magíster en Gerencia de Empresas. Doctora en Ciencias Gerenciales. Post-Doctora en Ciencias Gerenciales. Profesora e Investigadora de LUZ. Núcleo Punto Fijo. E-mail: romenarvaez@cantv.net

** Lic. en Contaduría Pública. Magíster en Gerencia de Empresas. Doctorante en Planificación y Gestión del Desarrollo Regional. Profesora e Investigadora de LUZ Núcleo Punto Fijo.

E-mail: hurtadoj@cantv.net 
Estrategias competitivas para fortalecer sectores de actividad empresarial...

Narváez, Mercy y Fernández, Gladys

they reside face new challenges and adapt to the changes that such a situation supposes. This article intends to identify some competitive strategies that would allow managerial sectors to obtain an advantageous position in the world network. Starting from a descriptive analysis based on theoretical contrasting, this study indicates that to reach a competitive position in the global market, new schemes of action must be established, such as: integrating behavior, development of central capacities, cooperation with external entities, specialization, solidarity and social contribution.

Key words: Competitive strategies, global market, managerial activity sectors, competitiveness.

\section{Introducción}

El concepto de estrategia competitiva ha venido evolucionando a lo largo del tiempo. En un primer momento se presentó el modelo planteado por Porter (1986), el cual se constituye en el paradigma base de este tipo de estrategia, en este modelo el autor establece las llamadas estrategias genéricas, en las cuales las empresas pueden conseguir ventajas competitivas mediante los costos bajos o el liderazgo en costos, es decir, participar en el mercado con una ventaja en costos que sea atractiva a los clientes; la diferenciación, a través de la incorporación de atributos tangibles o intangibles, los cuales permiten que el producto sea percibido por los clientes como único; y mediante la estrategia de alta segmentación, enfocándose en un grupo de compradores en particular, en un segmento de la línea de producto o en un mercado geográfico.

De igual manera Francés (2001) plantea el Paradigma de la Coopetencia, el cual se fundamenta en combinar la competencia entre empresas a través de la cooperación. El punto de partida para una estrategia basada en la coopetencia es pensar en los complementos, es decir, la interdependencia entre diferentes actores para la creación y desarrollo de mercados y competir para repartirlos.
Posteriormente, se plantea el modelo denominado "Designio Estratégico", la fundamentación teórica del mismo establece: competir por el futuro, más que por el presente. El Designio Estratégico reconoce que las empresas no compiten únicamente dentro de las fronteras de las industrias existentes, sino también por la conformación futura de las industrias.

Por otra parte, Azua y Andersen (2000) señalan un nuevo paradigma llamado: El Polinomio Competitivo = Busi-

ness + Government + Community, en este modelo se plantea un sistema integrador de una serie de elementos interdependientes, sus relaciones, sus condicionantes y aceleradores. Este enfoque busca que se generen plataformas económicas competitivas potenciadoras y receptoras de la interrelación entre la Administración Pública y Gobiernos comprometidos en un proceso estratégico largo - placista de competitividad y bienestar.

El Polinomio Business + Government + Community hacia la competitividad muestra la interacción que debe existir entre el sector empresarial (empresas e industrias), los gobiernos y la comunidad, constituyendo una base responsable de la atracción / generación de competitividad y bienestar para el conjunto de los clientes-ciudadanos que bajo su ámbito de interés, se implican en el desarrollo de una nueva sociedad a partir de nue- 
vos contratos y relaciones (Azua y Andersen, 2000).

Ahora bien, la evolución que se ha venido generando en el concepto de estrategia competitiva desde la formulación hecha por Porter hasta los nuevos paradigmas establecidos hoy día en los cuales se han venido incorporando diferentes elementos, modifica la manera de definir, explicar y más aún aplicar el modelo de competitividad, sobre todo si se tiene certeza de la existencia e interrelación de múltiples factores propios de un entorno dinámico, cambiante y turbulento que cada vez más condicionan y configuran una nueva forma de conducirse en el mundo empresarial.

Todo lo antes planteado implica modos diferentes de comportamiento, medios, técnicas y modelos empresariales distintos que faciliten el desarrollo de competencias y condiciones para responder a las exigencias del entorno y de un mercado que ahora se establece a escala mundial. En tal sentido, este artículo persigue identificar algunas estrategias que permitan a las empresas y los sectores de actividad donde estas se encuentran mejorar su posición competitiva con el fin de conquistar y mantener un espacio en el entramado mundial.

\section{La Globalización y su impacto en las organizaciones empresariales}

La globalización es un proceso que se ha venido experimentando desde hace más de dos décadas y el mismo puede ser definido como un proceso político, económico, social y ecológico que tiene lugar en todo el planeta, en el cual cada vez hay una mayor interrelación e interdependencia entre las personas y las organizaciones del mundo. Para que este proceso haya ocurrido es indudable el significativo papel que ha jugado el avance en los sistemas tecnológicos tales como la informática y las telecomunicaciones, y la implantación de innovaciones radicales como microelectrónica, biotecnología, ingeniería genética, nuevos materiales, nuevas formas y técnicas de organización.

Dentro de dicho proceso de globalización se encuentra la llamada "globalización económica", la cual ocurre mediante el intercambio de bienes y servicios entre los mercados de diferentes países. Al respecto, la Federación Luterana Mundial FLM (2002) define este proceso como un proyecto que conduce a la economía mundial a una dirección específica, fundamentado en la teoría neoliberal y que coloca como prioridad el libre movimiento del capital de inversión, la maximización de las ganancias y el crecimiento, además conduce a una creciente dependencia de las fuerzas del mercado.

Por otra parte, Gamboa et al (2001), señalan que desde finales del siglo XX, los avances ocurridos en campos como la informática, la biotecnología, la robótica, la microelectrónica, las telecomunicaciones y el transporte, conforman el surgimiento de un nuevo paradigma tecnológico, incrementa el flujo de información y mercancías del planeta y determinan nuevas creencias y posibilidades de producir bienes y servicios a más bajos costos y distribuirlos a escala mundial, generando de esta manera la conformación de la llamada "aldea global", globalización, mundialización de la economía o 
Estrategias competitivas para fortalecer sectores de actividad empresarial...

Narváez, Mercy y Fernández, Gladys

economía informacional y global, que profundiza la interconexión e interdependencia, por su amplitud y velocidad afecta a individuos, empresas o naciones.

Tal situación ha originado un cambio radical en la economía mundial. Según expresa Kotler y Armstrong (1998), hoy en día casi todas las empresas (grandes o pequeñas), se han visto afectadas de alguna manera por la competencia global. En la actualidad las empresas no solo están tratando de vender un mayor número de bienes producidos localmente en los mercados internacionales, sino que están comprando componentes y suministros en el extranjero para la generación de sus bienes y servicios.

En tal sentido, se puede afirmar que la globalización como proceso impacta de forma determinante la manera de comportarse de cualquier organización empresarial, pues esta debe tratar de al menos sobrevivir elaborando productos que estén dentro de los parámetros de las empresas que liderizan los mercados. En este contexto, de acuerdo a Ferrer y Clemenza (2006), las organizaciones deben actuar más allá del sistema tradicional de gestión, buscando crear factores de diferenciación sostenibles en el tiempo para de esta manera distinguirse de otras empresas y a la vez obtener ventajas competitivas.

En concordancia con lo anteriormente planteado Drucker (1999) señala que la empresa con un desempeño inferior a los parámetros más altos del mundo causa atrofia, aunque los costos de producción sean muy bajos y los subsidios oficiales sean muy altos. Esta situación aplica a todas las áreas de la empresa: di- seño, marketing, finanzas, innovación y la administración en su totalidad.

Toda empresa aún cuando sea local está situada en el contexto de la economía mundial y al respecto debe reorganizarse tanto a nivel interno como dentro de su entorno inmediato para al menos tratar de nivelarse con los estándares de las empresas líderes. Al efecto no bastan los cambios incrementales como la automatización intensiva y la creación de redes informáticas, pues lo que se busca es la consecución simultánea de eficiencia, flexibilidad, calidad y velocidad de reacción.

Lo antes expresado, conduce a estructurar una nueva forma de organización empresarial, la cual según señalan Siliceo et al. (1999) sea capaz de crear ambientes globalizados, con criterios y normas que puedan hacerla funcionar en cualquier país del mundo en la creación de productos y servicios de calidad, competitivos, en continuo desarrollo y perfeccionamiento. Una nueva organización donde los estándares de calidad, de diseño y estructura organizacional tienden a homogeneizarse y formar una cultura de negocios internacional, de clase mundial globalizada.

\section{La Competitividad y el desarrollo de Sectores de Actividad Empresarial}

El desarrollo económico es un proceso evolutivo cuyas expresiones y resultados dependen de la interacción de un conjunto de factores con las capacidades productivas del territorio, lo cual determina el grado de competitividad en un entorno caracterizado por una creciente interdependencia global. Si bien el proceso de 
globalización ha intensificado el flujo de factores de la producción y construido patrones de producción industrial y distribución comercial cada vez más globales, los sistemas económicos y sociales en el ámbito local-regional tienen la posibilidad de definir su papel con respecto a la dinámica del desarrollo, haciendo frente a los retos del proceso antes mencionado (Woo, 2004).

Para ser competitivo en un contexto de crecientes interdependencias se requiere contar con ciertos elementos tales como: la productividad, el conocimiento, las habilidades, la inteligencia económica y de mercadeo, la calidad de producto /servicio, la producción para nichos especializados del mercado, las capacidades gerenciales y organizacionales, la flexibilidad en la producción, el desarrollo tecnológico, la infraestructura física y social, la solidez institucional, entre otros, los cuales determinan la capacidad de competencia de un sistema económico social (Woo, 2004). En tal sentido, cabe destacar que la competitividad se refiere a la capacidad de las unidades productivas -empresa, sectores, regiones o nacionespara hacer frente a sus competidores en los mercados nacionales e internacionales (Francés, 1997).

Sin embargo, se debe señalar que el núcleo de la competitividad se inicia en la empresa, la cual dirige sus objetivos hacia la generación de riqueza en términos de beneficios, reducción de costos, la innovación y la diferenciación. Al ser competitiva la empresa, esta influye en el sector industrial al cual pertenece, no obstante, para que un sector empresarial o industrial sea competitivo tiene que po- seer ciertas condiciones que potencien sus capacidades y permitan su permanencia en el largo plazo.

Según expresa Francés (2002), los sectores empresariales están constituidos por un conjunto de empresas y la competitividad del mismo se mide por la capacidad que tiene para colocar exitosamente sus productos en el mercado internacional en condiciones de libre competencia. Los sectores pueden considerarse de forma amplia, incluyendo organizaciones educativas, de investigación y de servicios vinculados a las empresas.

Por otra parte, desde la perspectiva del concepto de competitividad sistémica, la cual se caracteriza y distingue por reconocer que para alcanzar un desarroIlo industrial exitoso se requiere no solo una función de producción en el nivel micro, o de condiciones económicas estables en el nivel macro, sino también por la existencia de medidas del gobierno y organizaciones privadas de desarrollo orientadas a fortalecer la competitividad de las empresas (nivel meso). Además, la capacidad de vincular las políticas meso y macro está en función de un conjunto de estructuras políticas y económicas, de un conjunto de factores socioculturales y patrones básicos de organización (nivel meta) (CEPAL, 2001).

Según plantea la Comisión Económica para la América Latina y el Caribe la competitividad (2001) es sistémica por tres razones:

a) Una empresa en general no es competitiva por sí misma, especialmente si no cuenta con un centro de apoyo de proveedores, o servicios orientados a la producción, o una presión competitiva de competidores locales. 
Estrategias competitivas para fortalecer sectores de actividad empresarial...

Narváez, Mercy y Fernández, Gladys

La competitividad en el nivel micro está basada en la interacción. El aprendizaje por interacción (learning-by-interacting) es clave en el proceso de innovación, especialmente cuando se constituyen ventajas competitivas dinámicas.

b) Un entorno que favorece la competitividad se encuentra arraigado en un sistema nacional de normas, reglas, valores e instituciones que definen los incentivos que moldean el comportamiento de las empresas.

c) El estado tiene un papel decisivo en el momento de definir el desarrollo industrial y la restructuración productiva del país, especialmente bajo las nuevas modalidades de gobernabilidad.

Derivado de lo anterior se desprende que la competitividad de las empresas y de los sectores industriales de los cuales estas forman parte, viene dada por la calidad de las relaciones generadas entre ellas y las distintas organizaciones de su entorno. Es decir, por la creación de entramados empresariales articulados con el estado y el resto de la sociedad, gestionados de manera estratégica para optimizar algunas competencias y crear ventajas orientadas a lograr el desarrollo económico sostenible.

En términos internacionales se habla de competitividad en dos ámbitos: la nación y las organizaciones. La competitividad de una nación se refiere a la habilidad para crear y sostener a largo plazo, el valor económico agregado en relación con sus competidores (Granell, 1994). Para que una nación sea realmente competitiva se requiere que sus empresas hayan pasado por un profundo cambio es- tructural y se hayan adaptado a las nuevas condiciones de un mercado global en correspondencia con el avance científico y técnico ocurrido en el tiempo.

Gabiña (1999) señala que en algunas naciones el apoyo a la modernización de las empresas es una de las políticas más importantes que se persiguen. En dichas naciones, la política industrial institucional está comprometida con los diversos actores económicos en fomentar e impulsar temas relacionados con las mejoras de la calidad y diseño, con el incremento de los gastos en Investigación y Desarrollo (I+D), con la elaboración de planes de formación permanente, entre otros, todo esto con el objeto de generar procesos de adaptación y mejora de la competitividad.

Este mismo autor refiere que las nuevas claves de competitividad en las naciones se centran en factores intangibles o materiales. El nivel de las competencias, habilidades y conocimientos, la capacidad organizativa, los niveles de cualificación de los trabajadores, la adhesión de las voluntades a los proyectos a largo plazo, la dirección y el análisis estratégico son, entre otros los principales elementos diferenciadores de unas naciones con respecto a otras. Dichos factores a mediano plazo, serán los que marcarán las diferencias en el desarrollo económico de las diferentes naciones.

\section{Estrategias Competitivas en el contexto del mercado global}

Para que las empresas puedan ingresar y alcanzar una posición ventajosa en el mercado global se requiere que éstas posean múltiples habilidades y mu- 
chas capacidades que les permitan enfrentar la hipercompetencia que se vive en el proceso de globalización. Además, se requiere por parte de los gerentes la formulación e implementación de estrategias empresariales que permitan dar respuesta a los desafíos del entorno interno y externo en el mediano y largo plazo.

Según Mintzberg et al. (1997) la estrategia empresarial se define como un plan que integra las principales metas y políticas de una organización y, a la vez, establece la secuencia coherente de las acciones a realizar. Una buena estrategia ayuda a poner orden y asignar, con base tanto en sus atributos como en sus deficiencias internas, los recursos de una organización con el fin, de lograr una situación viable y original, así como anticipar los posibles cambios en el entorno y las acciones imprevistas de los oponentes inteligentes.

Dentro de las estrategias empresariales están las llamadas estrategias competitivas, las cuales según Porter $(1986,1999)$ buscan posicionar a la empresa para maximizar el valor de las capacidades que la distinguen de sus competidores. La estrategia competitiva significa elegir deliberadamente un conjunto de actividades diferentes para prestar una combinación única de valor. Sin embargo, hoy en día ya no es suficiente formular estrategias para ser competitivo solo a nivel de la empresa, mediante la construcción de una cadena de valor para la misma, pues cada vez se hace más necesaria la conformación de sectores que permitan la interacción conjunta y el fortalecimiento de sus empresas, propiciando la libre competencia, pero además favoreciendo la articulación productiva a tra- vés de cadenas empresariales y asociaciones, y como entes de enlace eficaz ante los gobiernos, promoviendo de esta manera la permanencia de las empresas que los conforman en el largo plazo.

Por ello, ser competitivo en un mercado global implica un proceso paulatino, el desbordar lecturas atomizadas y particulares sobre la empresa, el sector, el país hacia una lectura sistémica que facilite el encadenamiento armonioso de estructuras políticas, financieras, de producción, y mercados, soportadas por nuevas instituciones normativas y políticas, facilitando así la articulación de estas unidades, para hacer frente a la dimensión global con base en las ventajas competitivas particulares del contexto (Benavides, 2002).

En tal sentido se requiere entonces de la identificación de algunas estrategias que sirvan para potenciar el desarrollo de sectores de actividad y a su vez promuevan el crecimiento económico y la capacidad competitiva de las regiones y por ende de los países. Entre estas estrategias se destacan las siguientes:

- Integración y cooperación, los empresarios deben asumir que para competir en el mercado mundial no pueden actuar de manera descentralizada y aislada, sino en forma de clusters industriales (grupo de empresas organizados en redes de colaboración), todo esto con el fin de hacer frente a los requerimientos del entorno y generar efectos acumulativos de aprendizaje e innovación durante la interacción que ocurra entre las empresas (Esser et al., 1996). Esta manera de integración busca que muchas empresas se adapten al nuevo cosmopolitismo de la economía global, conjugando conoci- 
Estrategias competitivas para fortalecer sectores de actividad empresarial...

Narváez, Mercy y Fernández, Gladys

miento, competencia y conectividad, a la vez que se proporcionen entre si la capacidad para atraer los recursos faltantes "lagunas" a través de la cooperación y de esta manera hacerse estratégicos en términos de competitividad (Azua y Andersen, 2000).

Para que el proceso de cooperación e integración entre empresas sectoriales sea exitoso se requiere la conformación de una política industrial activa que genere los mecanismos sociales de coordinación y apoyo a la acción individual mediante la concertación de los factores de producción, así como la participación de los gobiernos (nacional, estadales y municipales), y los organismos empresariales en cada región.

- Desarrollo de capacidades medulares, los gerentes-empresarios deben comprender que las empresas y los sectores empresariales son competitivos cuando poseen capacidades además de competencias que les permitan estar en procesos constantes de innovación en productos y procesos.

Al respecto señalan Ferrer y Pérez (2005) que las competencias medulares técnicas son la fuente más significativa de la diferenciación competitiva, dada su capacidad para generar productos y servicios con atributos únicos (diferenciación) valorados por los clientes. Igualmente las capacidades críticas pueden ser vistas como las funciones, las habilidades y los procesos de negocios que puedan tener un impacto crítico en la posición competitiva. La competencia medular existe cuando hay una capacidad o una combinación de capacidades primarias o críticas que proporcionan una ventaja de negocios real y sostenida.
En concordancia con lo antes planteado y trasladándolo a sectores de actividad empresarial, Villareal (2002) señala que la competitividad sectorial se basa en tres capitales fundamentales: El Capital Organizacional, El Capital Logístico y el Capital Intelectual, siendo este el nuevo factor de competitividad en la era del conocimiento y que requiere ir más allá del concepto tradicional de desarrollo científico y tecnológico, para enfocar éste en la capacidad creativa sistémica para promover la innovación apoyado en nuevos enfoques de educación que enfaticen el "aprender a aprender", el "aprender a emprender" y el "aprender haciendo" en los propios procesos del trabajo productivo.

- La especialización del sector, mientras más grande sea el mercado, más especializadas deben ser las empresas. Conforme el mundo transita hacia una economía global, las empresas van a tener que especializarse más y más (Ries, 1996). Tal situación lleva a las empresas a centrarse o enfocarse en sus actividades medulares con el fin de dominar en el mercado. Los sectores empresariales son poderosos cuando pueden adueñarse del mercado ofreciendo a sus clientes lo que ellos requieren de las empresas en mejores condiciones de calidad, eficiencia, costos, innovación, entre otros.

- Cooperación con entes externos al sector (Gobierno y Comunidad), el objetivo de todo gobierno es proporcionar riqueza y bienestar social a sus ciudadanos, incrementándolo a lo largo del tiempo. Por ello, el papel de éstos a favor de la competitividad pasan por su capacidad para facilitar el desarrollo de industrias y empresas en su ámbito territorial 
de responsabilidad, generando un clima adecuado y desarrollando políticas públicas en línea con necesidades específicas de las empresas y sectores empresariales (Azua y Andersen, 2000). Sin embargo, las empresas y los sectores empresariales también pueden contribuir a través de procesos de relación y cooperación con las diferentes organizaciones del estado en la tarea de fomentar una mejor calidad de vida a la comunidad en general e impulsar el progreso del marco regulativo para la implantación de nuevas empresas y sectores que contribuyan con el desarrollo de la sociedad.

- Fundamentar la competitividad y productividad del sector empresarial sobre la base de una mayor solidaridad y cooperación social, con el fin de lograr un crecimiento material del sector propiamente dicho y del entorno social donde este se encuentre ubicado. El éxito empresarial en el largo plazo depende del desarrollo económico y social del país donde se esté ubicado y tal desarrollo es en parte responsabilidad de las empresas que lo conforman. En tal sentido, las empresas deben crear condiciones que permitan satisfacer a los clientes, ofrecer productos de calidad, rendir cuentas a sus accionistas, cumplir con los proveedores, respetar a sus competidores, fomentar el bienestar de sus trabajadores, la sustentabilidad del medio ambiente y contribuir a mejorar las condiciones de vida de la comunidad a la cual pertenecen (Lemaresquier, 2004).

\section{Consideraciones Finales}

La interdependencia económica que existe entre las naciones y las organi- zaciones empresariales derivada del proceso de globalización crea la necesidad de que las empresas asuman un modelo industrial y productivo que se fundamente en la articulación de ellas a través de un encadenamiento armonioso de distintas estructuras apoyadas además por diferentes instituciones y organismos que les permitan hacer frente al entorno y obtener así algunas ventajas competitivas propias del contexto global.

Tal situación conduce a afirmar que para las empresas y los sectores de los cuales ellas forman parte sean competitivos y se ubiquen en una situación ventajosa en un mercado que cada día es más globalizado y donde la competencia se hace muy difícil, se requiere readaptarse a las condiciones del mercado mediante un profundo cambio estructural. De tal situación no escapa ninguna empresa ni sector empresarial, pues hasta las posiciones más hegemónicas son también vulnerables a esta nueva dimensión global.

El hecho de que una nación sea competitiva, comienza en sus unidades empresariales, las cuales deben readecuarse con el objeto de mejorar sus competencias medulares, sus costos y la velocidad para dar respuesta a las exigencias del mercado. Ahora bien, para lograr la competitividad internacional de un país se requiere cumplir con dos requisitos fundamentales: primero que las empresas realicen esfuerzos para mejorar sus productos, procesos y ser más eficientes, flexibles y rápidas para reaccionar a los cambios del entorno; y en segundo lugar, estar insertas en redes articuladas dentro de las cuales los esfuerzos realizados por las empresas se apoyen por todo un conjunto de servicios, instituciones e infraes- 
Estrategias competitivas para fortalecer sectores de actividad empresarial...

Narváez, Mercy y Fernández, Gladys

tructura que faciliten y promuevan el desarrollo de una plataforma económica que les permita hacer frente a la dimensión global.

Las estrategias empresariales expuestas en este trabajo, tales como: integración y cooperación empresarial, desarrollo de capacidades medulares, especialización, solidaridad y cooperación social, entre otras, permitirán de alguna manera potenciar el desarrollo de los sectores empresariales, buscando de esta forma que éstos se nivelen con los indicadores establecidos a escala mundial para posteriormente lograr su espacio en el mediano y largo plazo, a través de la creación de futuro, la construcción de escenarios factibles y el desarrollo de capacidades necesarias para dominar el mercado global.

\section{Referencias Bibliográficas}

Azua, Jon y Andersen, Arthur (2000). Alianzas Competitivas para la nueva Economía. Mc GrawHill. España.

Benavides, Olga (2002). Competencias y Competitividad. Diseño para organizaciones latinoamericanas. McGrawHill. Colombia.

CEPAL (Comisión Económica para América Latina y el Caribe) (2001). Elementos de competitividad sistémica de las pequeñas y medianas empresas (PYME) del Istmo Centroamericano. LC/Mex/L.499.8.

Drucker, Peter (1999). Los Desafíos de la Gerencia para el Siglo XXI. Grupo Editorial Norma. Colombia.

Esser, Klaus; Hillebrand, Wolfgang; Messner, Dirk y Meyer-Stamer, Jörg (1996). Competitividad Sistémica: Nuevo desafío a las empresas y a la política.
Revista de la CEPAL. No. 59. Págs: $39-52$.

Ferrer, Juliana y Clemenza, Caterina (2006). Habilidades gerenciales como fundamento de la estrategia competitiva en los sectores de actividad metalmecánica venezolana. Revista Tendencias. Vol VII № 1. Páginas: 81-100. Universidad de Nariño. Colombia.

Ferrer, Juliana y Pérez, Rosario (2005). Capacidades medulares en la búsqueda del éxito organizacional. Revista especializada Mercadeo unplugged. Año 2. Edición No. 9. Maracaibo. Venezuela.

FLM. Federación Luterana Mundial (2002). Llamado a participar en la transformación de la Globalización Económica. LWF Tenth Assembly. Canadá.

Francés, Antonio (1997). Zulia. Competitividad para el desarrollo. Ediciones IESA, C.A.

Francés, Antonio (2001). Estrategia para la empresa en América Latina. Ediciones IESA, C.A. Caracas.

Francés, Antonio (2002). ¿Cómo mejorar la competitividad de las empresas nacionales? Debates IESA. Vol. VII. No. 4 Abril - Junio 2002. Ediciones IESA. Caracas.

Gabiña, Juango (1999). Prospectiva y Planificación Territorial. Alfaomega Grupo Editor, S.A. Colombia.

Granell, Elena (1994). Recursos Humanos y Competitividad en Organizaciones Venezolanas. Ediciones IESA C.A. Caracas.

Gamboa, Teresa; Arellano, Madelein y Nava, Yuneska (2001). Estrategias Empresariales: Aproximación a una tipología. Revista Venezolana de Ciencias Sociales. UNERMB. Vol 5. No. 2. Págs: 132-154. Venezuela.

Kotler, Philip y Armstrong, Gary (1998). Fundamentos de Mercadotecnia. Pren- 
tice Hall Hispanoamericana, S.A. México.

Lemaresquier, Thierry (2004). La Ética Empresarial y la Competitividad. Disponible en: www.compite.org.mx/ eventos/2congreso-respsocial/ponencia/ponencia

Mintzberg, Henry; Brian, James y Voyer, John (1997). El Proceso Estratégico. Prentice Hall Hispanoamericana, S.A. México.

Porter, Michael (1986). Estrategia Competitiva. Técnicas para el Análisis de los Sectores Industriales y de la Competencia. Compañía Editorial Continental, S.A. México.

Porter, Michael (1999). Ser Competitivo. Ediciones Deusto S.A. España.

Ries, Al (1996). Enfoque. El único futuro de su empresa. McGrawHill. México.
Siliceo, Alfonso; Casares, David y González, José Luis (1999). Liderazgo, valores y cultura organizacional. McGrawHill. México.

Villarreal, René (2002). América Latina frente al reto de la Competitividad: Crecimiento con Innovación. Revista lberoamericana de Ciencia, Tecnología, Sociedad e Innovación. No. 4. Septiembre -Diciembre 2002. Disponible en: www.campus-oei.org/revistactsi/numero4/alaina.htlm

Woo Gómez L. Guillermo (2004). Descentralización y desarrollo económico local. La configuración de nuevos espacios territoriales del desarrollo económico latinoamericano. En: El desarrollo local en América Latina. Logros y desafíos para la cooperación europea. Editorial Nueva Sociedad. Caracas. 